\title{
FATORES ASSOCIADOS AO USO DE ANFETAMINAS ENTRE CAMINHONEIROS
}

\author{
Juliana Gusmão Fonseca ${ }^{1}$ \\ Gustavo Magalhães Viana ${ }^{2}$ \\ Joyce Elen Murça de Souza ${ }^{3}$ \\ Luiza Augusta Rosa Rossi-Barbosa ${ }^{4}$ \\ Recebido em: 19 mar. 2018 \\ Aceito em: 25 fev. 2019
}

\begin{abstract}
RESUMO: As anfetaminas conhecidas como "rebites" são normalmente utilizadas por caminhoneiros. Este estudo teve como objetivo verificar a prevalência e fatores associados à utilização autorrelatada de anfetaminas entre caminhoneiros que trafegam na rodovia BR 251, no trecho de Montes Claros, MG, com parada em um posto de combustível. Trata-se de um estudo transversal, quantitativo com seleção dos indivíduos por amostragem de conveniência. Foi utilizado um questionário com dados sociodemográficos, econômicos, ocupacionais e relacionados às anfetaminas. Realizou-se a análise bivariada, cuja variável dependente foi o uso de anfetaminas e aquelas que apresentaram associação ao nível de $20 \%(p \leq 0,20)$ foram selecionadas para a análise múltipla utilizando a Regressão de Poisson. Permaneceram no modelo as variáveis com desfecho ao nível de $5 \%(p \leq 0,05)$. Dentre os 306 pesquisados, 22,2\% eram usuários de anfetaminas, sendo o princípio ativo mais utilizado o Femproporex (Desobesi®). A média de idade foi de 41 anos, variando de 22 a 77 anos. A maioria trabalha mais de 10 horas diárias. O uso de anfetaminas esteve associado aos profissionais mais jovens e com maior carga horária de trabalho. Faz-se necessário um controle maior sobre a venda desses medicamentos por parte dos órgãos competentes.
\end{abstract}

Palavras-chave: Prevalência. Anfetaminas. Drogas llícitas.

\section{FACTORS ASSOCIATED WITH THE AMPHETAMINES USE AMONG TRUCK DRIVERS}

ABSTRACT: Amphetamines known as "rivets" are commonly used by truck drivers. This study aimed to verify the prevalence and factors associated with the self-reported use of amphetamines among truck drivers who travel on the BR 251 highway, in the Montes Claros stretch, MG, stopping at a fuel station. It is a cross-sectional, quantitative study with selection of individuals by convenience sampling. A questionnaire with socio-demographic, economic, occupational and amphetaminerelated data was used. The bivariate analysis was performed, whose dependent variable was the use of amphetamines, and those that showed association at the level of $20 \%(p \leq 0.20)$ were selected to multiple variety analysis using Poisson Regression. Remain in analysis only variables whose end point was $5 \%(p \leq 0.05)$. Among 306 participants, $22.2 \%$ had used amphetamine, Femproporex (Desobesii))was the most common active principle used. Mean age was 41 years, range 22 to 77 years old. Most interviewed works more than 10 hours a day. Younger drivers and more daily hours of work were associated with amphetamine use. Finally, to decrease amphetamine use and abuse, it is essential a closer sale control on this drugs by the government.

Keywords: Prevalence. Amphetamines. Illicit Drugs.

\footnotetext{
1 Faculdades Unidas do Norte de Minas - Funorte.

2 Faculdades Unidas do Norte de Minas - Funorte.

3 Universidade Estadual de Montes Claros - Unimontes.

4 Universidade Estadual de Montes Claros - Unimontes.
} 


\section{INTRODUÇÃO}

As anfetaminas, conhecidas como "rebites", são potentes estimulantes do Sistema Nervoso Central (SNC) e vêm sendo usadas com maior ocorrência entre os caminhoneiros devido à necessidade de se manterem acordados (TAKITANE et al., 2013). Seu uso indiscriminado, sem as devidas orientações e prescrições médicas, é considerado um sério problema de saúde pública (NASCIMENTO, NASCIMENTO, 2007).

Após ingestão, as anfetaminas são absorvidas rapidamente pelo trato gastrointestinal, passando livremente pela barreira hematoencefálica, causando efeitos pronunciados sobre o sistema nervoso central. Também são absorvidas pela mucosa nasal e distribuídas na maior parte dos tecidos. Os principais efeitos centrais da substância estão a estimulação locomotora, euforia, excitação, anorexia (MARCON et al., 2012). Tais efeitos graves e residuais fazem com que o condutor se depare com situações de risco nas estradas, impedindo-o de viajar em segurança (NASCIMENTO, NASCIMENTO, 2007).

No Brasil, alguns estudos têm demonstrado que os motoristas de caminhão se apresentam vulneráveis ao uso de substâncias psicoativas (SPA) e este comportamento é empregado como forma de compensar as condições inadequadas de trabalho: longas viagens, pressa para chegar ao destino, baixas remunerações, condições de saúde e de vida no geral (LEYTON et al., 2012; SINAGAWA, et al., 2015) tendo, também, a finalidade de reduzir o cansaço e melhorar o rendimento no trabalho e a produtividade (SILVAJÚNIOR et al., 2009). Em um desses estudos desenvolvido no Brasil pode-se verificar que mais de $27 \%$ dos caminhoneiros já foram surpreendidos e já estiveram envolvidos em acidentes de trânsito decorrentes do uso destas drogas (NASCIMENTO, NASCIMENTO, 2007).

As anfetaminas deixam o usuário em estado de alerta, tornando preocupante seu uso no trânsito, isto porque elas aumentam a autoconfiança, predispondo o motorista a situações de risco. Posteriormente, a fadiga e sonolência substituem o efeito estimulante, o que deixa o condutor ainda mais suscetível a situações negativas no trânsito (LEYTON et al., 2012).

Este artigo teve como objetivo verificar a prevalência autorrelatada do uso de anfetaminas e os fatores associados ao seu uso entre caminhoneiros que trafegam na rodovia BR 251, com parada em um posto de combustível na altura do $\mathrm{Km} 9,78$ no norte de Minas Gerais.

\section{MATERIAL E MÉTODOS}

Este estudo teve um caráter epidemiológico de delineamento transversal. Participaram 306 caminhoneiros que frequentaram um dos postos de combustíveis situado na rodovia BR 251. Este foi escolhido por estar na entrada do município de Montes Claros, MG, ter um grande fluxo de caminhoneiros onde param para fazer refeições e descansar 
como, também, pelo fato de um dos pesquisadores ter trabalhado no local.

Montes Claros, MG é a cidade polo da região norte-mineira com população estimada em 402.027 mil segundo o Instituto Brasileiro de Geografia e Estatística (IBGE, 2017) e é o segundo maior entroncamento rodoviário do país, sendo rota de muitos caminhoneiros (MONTES CLAROS, MG, 2017).

A seleção dos indivíduos foi por amostragem de conveniência. Estes eram abordados tendo como incentivo a aferição da pressão e posteriormente respondiam o questionário sobre o uso de anfetaminas, porém cerca de $10 \%$ recusaram responder. 0 questionário foi elaborado a partir de pesquisas anteriores (NASCIMENTO, NASCIMENTO, 2007; OLIVEIRA et al., 2013; TAKITANE et al., 2013) no qual foram abordados os seguintes parâmetros: sexo, idade, escolaridade, renda mensal, carga horária de trabalho, tempo de trabalho, se envolveu em acidente na rodovia, se dormiu ou cochilou na direção, e aquelas relacionadas ao uso de anfetaminas (como adquiriu, se usa com frequência, em que casos usa, em que casos não usa, se sente algum mal-estar após o uso, se recebeu orientações quanto ao uso, se conhece os problemas quanto ao uso, como informou sobre as anfetaminas, se acha necessário receber orientações).

Após concordarem participar da pesquisa, os caminhoneiros assinaram o Termo de Consentimento Livre e Esclarecido. O preenchimento do questionário foi realizado pelos pesquisadores. Este trabalho foi realizado durante uma semana, no período da manhã no mês de outubro de 2014.

Após o processo de coleta, os dados foram tabulados e analisados no programa Predictiv Analytics Software (PASW®STATISTIC) versão 18.0. Primeiramente, foi realizada a análise descritiva com verificação das frequências relativas e absolutas, medidas de tendência central e de dispersão. Posteriormente procedeu-se a análise bivariada por meio do teste qui-quadrado de Pearson entre a variável desfecho, uso de anfetaminas, e as variáveis independentes dicotomizadas. Aquelas que apresentaram associação até o nível de significância estatística de $20 \%(p \leq 0,20)$ foram selecionadas para a análise múltipla utilizando a Regressão de Poisson (stepwise forward) para verificar a razão de prevalência do uso de anfetaminas. Permaneceram no modelo as variáveis com desfecho ao nível de $5 \%(p \leq 0,05)$.

O projeto foi submetido ao Comitê de Ética em Pesquisa das Faculdades Unidas do Norte de Minas - Funorte e aprovado sob o número 881.327.

\section{RESULTADOS}

A média de idade dos 306 respondentes foi de 41 anos, (DP $\pm 10,5)$ sendo a idade mínima de 22 e máxima de 77 anos. A faixa etária mais prevalente foi dos 30 aos 39 anos. A maioria tem o ensino fundamental, mas observa-se que há indivíduos com nível superior nesta profissão. Grande parte trabalha entre 11 a 14 horas e estão inseridos há menos de 10 anos como caminhoneiros, sendo que $22,9 \%$ referiram trabalhar entre seis a dez anos. 
Os demais dados sociodemográficos, econômicos e de trabalho se encontram na tabela 1.

Tabela 1. Dados sociodemográficos, econômicos, ocupacionais e riscos entre os caminhoneiros. Montes Claros, MG, 2014.

\begin{tabular}{|c|c|c|}
\hline Variáveis & $\mathrm{n}=306$ & $\%$ \\
\hline \multicolumn{3}{|l|}{ Sexo } \\
\hline Masculino & 302 & 98,7 \\
\hline Feminino & 4 & 1,3 \\
\hline \multicolumn{3}{|l|}{ Faixa etária (anos) } \\
\hline$\leq 29$ & 36 & 11,8 \\
\hline $30-39$ & 105 & 34,3 \\
\hline $40-49$ & 88 & 28,8 \\
\hline $50-59$ & 56 & 18,3 \\
\hline $60-69$ & 19 & 6,2 \\
\hline$\geq 70$ & 2 & 0,6 \\
\hline \multicolumn{3}{|l|}{ Escolaridade } \\
\hline Analfabeto & 6 & 2,0 \\
\hline Ensino Fundamental & 166 & 54,2 \\
\hline Ensino Médio & 127 & 41,5 \\
\hline Ensino Superior & 7 & 2,3 \\
\hline \multicolumn{3}{|l|}{ Renda mensal * } \\
\hline Até 3 salários & 65 & 21,2 \\
\hline Mais de 3 salários & 241 & 78,8 \\
\hline \multicolumn{3}{|c|}{ Carga horária de trabalho / dia } \\
\hline De 4 a 6 horas & 6 & 2,0 \\
\hline De 7 a 10 horas & 88 & 28,8 \\
\hline De 11 a 14 horas & 101 & 33,0 \\
\hline De 15 a 18 horas & 51 & 16,7 \\
\hline Mais de 18 horas & 60 & 19,6 \\
\hline \multicolumn{3}{|l|}{ Tempo de trabalho } \\
\hline Até 10 anos & 108 & 35,3 \\
\hline De 11 a 20 anos & 97 & 31,7 \\
\hline De 21 a 30 anos & 51 & 16,7 \\
\hline Mais de 30 anos & 50 & 16,3 \\
\hline \multicolumn{3}{|c|}{ Envolveu-se em algum acidente em rodovias } \\
\hline Sim & 102 & 33,3 \\
\hline Não & 204 & 66,7 \\
\hline \multicolumn{3}{|c|}{ Cochilou/dormiu na direção } \\
\hline Sim & 136 & \\
\hline Não & 170 & 55,6 \\
\hline
\end{tabular}

* Salário referência $=\mathrm{R} \$ 724,00$.

Dentre os caminhoneiros pesquisados $22,2 \%$ usam anfetaminas (Figura 1), sendo o Nobésio forte o mais utilizado, seguido do Desobesi-M cujo princípio ativo de ambos é o Femproporex. 
Imagem 1- Distribuição dos caminhoneiros $(n=306)$ sobre o uso de anfetaminas. Montes Claros, MG. 2014.

\section{Anfetaminas}



A tabela 2 apresenta os dados sobre a aquisição, frequência e os problemas quanto ao uso das anfetaminas entre os 68 caminhoneiros que afirmaram serem usuários. Verificou-se que a maioria usa os "ribites" quando começa a sentir sono, conhece os problemas quanto ao uso, sentem algum mal estar após o uso (arritmia, tontura, dor de cabeça, tremedeira dos membros, dor no peito), porém relataram necessário receber orientações a respeito das anfetaminas.

Tabela 2. Aquisição, frequência e problemas decorrentes do uso de anfetaminas por caminhoneiros. Montes Claros, MG, 2014.

\begin{tabular}{lll}
\hline Variáveis & $\mathrm{n}=68$ & $\%$ \\
\hline Como adquiriu a anfetamina & 63 & 92,6 \\
Amigo & 4 & 5,9 \\
Empresa & 1 & 1,5 \\
Parente & 37 & 54,4 \\
Se usa com frequência & 31 & 45,6 \\
Sim & & 45,6 \\
Não & 31 & 30,9 \\
Qual frequência & 21 & 23,5 \\
Raramente & 16 & 80,9 \\
Viagens noturnas & & 19,1 \\
Todas as viagens & 55 & 76,5 \\
Quando usa & 13 & 22,1 \\
Quando começa sentir sono & & 1,5 \\
Quando sente cansaço & 52 & \\
Quando não usa & 15 & 69,1 \\
Quando cumpre a rota & 1 & 30,9 \\
Quando as viagens são curtas & & \\
Quando sente mal & 47 & 98,5 \\
Algum mal estar após o uso & 41 & \\
Não & & 76,5 \\
Sim & & \\
Se recebeu orientação quanto ao uso & 67 & \\
Sim & 1 & \\
Se conhece os problemas quanto ao uso & & \\
Sim & 52 & \\
& &
\end{tabular}


Não

16

Como se informou sobre as anfetaminas

Amigos

Empresa

Médico

Parente

Farmacêutico

Se acha necessário receber orientações

Sim

Não
23,5

83,8

5,9

4,4

4,4

1,5

67,6

32,4

A análise bivariada com $p \leq 0,20$ mostrou associação entre uso de anfetaminas e idade, horas trabalhadas no dia, tempo de trabalho como caminhoneiro, envolvimento em acidentes na estrada e ter conhecimento dos problemas acerca do uso de tais substâncias (Tabela 3).

Tabela 3- Análise bivariada entre o uso de anfetaminas autorreferido por caminhoneiros e as variáveis sociodemográficas, econômicas, ocupacionais e riscos. Montes Claros, MG, 2014.

\begin{tabular}{|c|c|c|c|}
\hline \multirow[t]{2}{*}{ Variáveis } & \multicolumn{2}{|c|}{ Uso de anfetaminas } & \multirow{2}{*}{$\begin{array}{l}\text { Valor } p \\
p\end{array}$} \\
\hline & $\begin{array}{l}\text { Não } \\
\text { n (\%) }\end{array}$ & $\begin{array}{l}\text { Sim } \\
\mathrm{n}(\%)\end{array}$ & \\
\hline \multicolumn{4}{|l|}{ Sexo } \\
\hline Masculino & $236(78,1)$ & $66(21,9)$ & \multirow[t]{2}{*}{0,179} \\
\hline Feminino & $2(50,0)$ & $2(50,0)$ & \\
\hline \multicolumn{4}{|l|}{ Idade } \\
\hline$<39$ anos & $95(67,4)$ & $46(32,6)$ & \multirow[t]{2}{*}{0,000} \\
\hline$\geq 40$ anos & $143(86,7)$ & $22(13,3)$ & \\
\hline \multicolumn{4}{|l|}{ Escolaridade } \\
\hline Analfabeto/ensino fundamental & $131(76,2)$ & $41(23,8)$ & \multirow{2}{*}{0,441} \\
\hline Ensino médio/ensino superior & $107(79,9)$ & $27(20,1)$ & \\
\hline \multicolumn{4}{|l|}{ Renda mensal* } \\
\hline$\leq 3$ salários mínimos & $53(81,5)$ & $12(18,5)$ & \multirow[t]{2}{*}{0,411} \\
\hline >3 salários mínimos & $185(76,8)$ & $56(23,2)$ & \\
\hline \multicolumn{4}{|l|}{ Carga horária de trabalho } \\
\hline$\leq 14$ horas & $166(85,1)$ & $29(14,9)$ & \multirow{3}{*}{0,000} \\
\hline$>14$ horas & $72(64,9)$ & $39(35,1)$ & \\
\hline \multicolumn{3}{|l|}{ Tempo de trabalho } & \\
\hline$\leq 15$ anos & $109(71,7)$ & $43(28,3)$ & \multirow[t]{2}{*}{0,011} \\
\hline$>15$ anos & $129(83,8)$ & $25(16,2)$ & \\
\hline \multicolumn{4}{|c|}{ Envolveu-se em algum acidente em rodovias } \\
\hline Sim & $74(72,5)$ & $28(27,5)$ & \multirow{2}{*}{0,120} \\
\hline Não & $164(80,4)$ & $40(19,6)$ & \\
\hline \multicolumn{4}{|l|}{ Cochilou/dormiu na direção } \\
\hline Sim & $106(77,9)$ & $30(22,1)$ & \multirow[t]{2}{*}{0,951} \\
\hline Não & $132(77,6)$ & $38(22,4)$ & \\
\hline
\end{tabular}

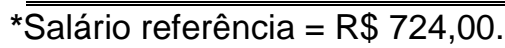

As variáveis que apresentaram significância com o desfecho ao nível de $5 \%$ $(p \leq 0,05)$ se encontram na Tabela 4 . Observa-se que a faixa etária mais jovem e jornadas de trabalho mais longas mantiveram no modelo final. 
Tabela 4- Resultado da Razão de Prevalência bruta e ajustada referente ao uso de anfetaminas pelos caminhoneiros. Montes Claros, Minas Gerais, Dezembro de 2014.

\begin{tabular}{lllll}
\hline \hline Variáveis & $\begin{array}{l}\text { RP bruto } \\
\text { (IC 95\%) }\end{array}$ & Valor-p & $\begin{array}{l}\text { RP ajustado } \\
\text { (IC 95\%) }\end{array}$ & Valor-p \\
\hline $\begin{array}{l}\text { Idade } \\
\quad 39 \text { anos }\end{array}$ & 1,00 & & 1,00 & \\
$\quad \begin{array}{l}\text { 240 anos } \\
\text { Horas trabalhadas }\end{array}$ & $0,857(0,770-0,954)$ & 0,005 & $0,880(0,818-0,947)$ & 0,001 \\
$\begin{array}{c}\text { carga horária) } \\
\quad \leq 14 \text { horas }\end{array}$ & & & & \\
$\quad>14$ horas & 1,00 & & 1,00 & \\
\hline \hline
\end{tabular}

\section{DISCUSSÃO}

A prevalência de usuários de anfetaminas entre motoristas de caminhão foi similar a outro estudo realizado com 684 motoristas em três rodovias do estado de São Paulo (OLIVEIRA et al., 2015). Porém, foi acima de diversos outros estudos os quais apresentaram variação de 2,7\% a 12,4\% (KNAUTH et al., 2012; LEYTON et al., 2015; OLIVEIRA et al., 2013; OLIVEIRA et al., 2015; SINAGAWA et al., 2015; SOUZA, PAIVA, REIMÃO, 2005; TAKITANE et al., 2013), e abaixo da pesquisa de Passos - MG, cuja prevalência foi de 66,0\% (NASCIMENTO, NASCIMENTO, 2007).

Essa variabilidade encontrada quanto à prevalência refere-se, provavelmente, às diferenças metodológicas utilizadas nos estudos mencionados, tais como, número de indivíduos na amostra, avaliação quanto ao tempo de uso da anfetamina, a forma de coleta, local onde os motoristas foram abordados e pela maneira de como a pergunta foi formulada.

Em outubro de 2011, a Agência Nacional de Vigilância Sanitária (ANVISA) divulgou a Resolução de número 52 (Resolução da Diretoria Colegiada - RDC 52/2011), proibindo a produção, dispensação, importação, exportação, prescrição e uso dos sais e isômeros de Anfepramona, Femproporex e Mazindol, assim como de seus produtos de biotransformação (BRASIL, 2011). Porém, a prevalência de anfetaminas no presente estudo e de outros estudos brasileiros, realizados após a resolução, mostram a continuidade do uso entre caminhoneiros (OLIVEIRA et al., 2013; OLIVEIRA et al., 2015).

No que se refere às fontes pelas quais se obtêm as substâncias em questão, a principal forma de aquisição no presente estudo foi através dos amigos. (OLIVEIRA et al., 2013). Enquanto um estudo realizado em Ponta Grossa - PR, destacou a obtenção das drogas em postos de gasolina, borracharias, farmácias, restaurantes, lanchonetes, mercadinhos e no Paraguai (WENDLER, BUSATO, MIYOSHI, 2003). Devido ao baixo custo, as anfetaminas são facilmente obtidas sem receita médica nas estradas brasileiras (LEYTON et al., 2012).

No modelo final do presente estudo, o uso de anfetaminas esteve associado a motoristas mais jovens e com mais horas de trabalho. A associação do uso de anfetaminas a motoristas jovens também esteve presente em outros estudos (KNAUTH et al., 2012; OLIVEIRA et al., 2015; WILLIAMSON, 2007). A relação entre o uso de anfetaminas e idade 
provavelmente se deve a pouca experiência dos jovens como motoristas profissionais (OLIVEIRA et al., 2015). É um grupo considerado mais vulnerável em decorrência da dificuldade em suportar as pressões no trabalho (KNAUTH et al., 2012). Por não saberem lidar com as diversidades da profissão, procuram nas drogas a solução (WILLIAMSON, 2007).

Sobre a elevada carga de trabalho, autores da pesquisa realizada em cinco municípios do Rio Grande do Sul observaram que esta variável combinada com a ausência de oferta de serviços de saúde, de educação e poucas opções de lazer nos locais em que permanecem parados, propicia o consumo das anfetaminas para o motorista ficar acordado. Na pesquisa realizada nas estradas interestaduais do Estado de São Paulo os caminhoneiros confirmam este fato ao relatarem que ingerem essas drogas com o objetivo de ficarem acordados para a condução de longas jornadas (SINAGAWA et al., 2015). A carga horária elevada produz desgaste físico e emocional buscando a solução no consumo de anfetaminas (KNAUTH et al., 2012).

De acordo com os participantes do estudo, o uso das drogas está relacionado à obrigação de manterem-se acordados para atender as demandas de trabalho e cumprir os prazos de entrega exigidos. Dessa maneira, os motoristas ainda conseguem realizar viagens extras, com o objetivo de elevar o salário (SILVA et al., 2016).

Merece destaque o uso do Femproporex Desobesi® na presente pesquisa. Assim como nos demais estudos, foi o medicamento mais usado entre os participantes. Trata-se de um anorexígeno que tem como princípio ativo o cloridrato de femproporex que, assim como os demais derivados anfetamínicos, promove a estimulação do SNC e apresenta elevado potencial para causar dependência (CODY, VALTIER, 1996, TAKITANE et al., 2013; WENDLER, BUSATO, MIYOSHI, 2003).

Sendo assim, sugere-se que alguns comportamentos sejam modificados: 1) diminuição do tempo diário de trabalho; 2) elevação da quantidade de pausas para descanso durante a jornada ocupacional; 3) mais folgas aos finais de semana; 4) sono noturno, de qualidade e por mais tempo; 5) evitar trabalhar ou conservar-se acordado durante a noite. Através de ações educativas e políticas públicas, será possível conscientizar os empregadores, bem como os motoristas rodoviários, para melhorar o estilo de vida e possibilitar um deslocamento mais seguro (NARCISO, MELLO, 2017).

É necessário o fortalecimento, principalmente na proposição de outras ações que podem ser implementação com o objetivo de conscientização e possível redução do curso das anfetaminas.

Apesar dos importantes achados, os resultados do presente trabalho devem ser interpretados sob a luz de algumas limitações. Um aspecto a ser destacado diz respeito à detecção do uso de anfetaminas, a qual foi aferida por meio do autorrelato. A taxa de nãoresposta também pode ter subestimado o número de usuário de anfetaminas. Outra limitação refere ao fato de não ter pesquisado sobre as demais drogas ilícitas, como por exemplo o crack e a cocaína, bem como a ausência de exames laboratoriais, em especial 
a análise da urina de tais motoristas.

\section{CONSIDERAÇÕES FINAIS}

A prevalência de usuários de anfetaminas entre a amostra de caminhoneiros do presente estudo foi de $22,2 \%$ tendo como princípio ativo mais utilizado o fenproporex (Desobesi®). O uso de anfetaminas esteve associado aos profissionais mais jovens e com maior carga horária de trabalho.

Os resultados demonstraram uma realidade preocupante quanto ao uso indiscriminado de substâncias psicoativas. Embora a RDC n․ 52 (Brasil, 2011) proíba a venda de anfetaminas sem notificação de receita, a comercialização clandestina continua ocorrendo, sendo um sério problema para a saúde pública. É de extrema urgência um controle maior sobre a venda desses medicamentos por parte dos órgãos competentes.

\section{REFERÊNCIAS}

BRASIL, Agência Nacional de Vigilância Sanitária. Resolução RDC nํ52, de 6 de outubro de 2011. Proibição do uso das substancias anfepramona, femproporex e mazindol, seus sais e isômeros, bem como intermediários e medidas de controle da prescrição e dispensação de medicamentos que contenham a substância sibutramina, seus sais e isômeros, bem como intermediários e dá outras providências. Diário Oficial da União, $2011 ; 10$ de outubro.

CODY, John; VALTIER, Sandra. Detection of amphetamine following administration of fenproporex. Journal of Analytical Toxicology. v. 20, n. 6; p. 425-431, 2007. disponível em:

IBGE - Instituto brasileiro de geografia e estatística; 2017. Disponível em: https://www.ibge.gov.br/

KNAUTH, Daniela Riva. et al. Manter-se acordado: a vulnerabilidade dos caminhoneiros no Rio Grande do Sul. Rev Saúde Pública. v. 46, n. 5, p. 886-893, 2012. Disponível em: http://www.scielo.br/scielo.php?script=sci_arttext\&pid=S0034-89102012000500016

LEYTON, Vilma. et al. Amphetamine, cocaine and cannabinoids use among truck drivers on the roads in the State of Sao Paulo, Brazil. Forensic Sci Int. v. 215, n. 1-3, p. 25-27, 2012. Disponível em: https://www.ncbi.nlm.nih.gov/pubmed/21511416

MARCON, Carine. et al. Uso de anfetaminas e substâncias relacionadas na sociedade contemporânea. Disciplinarum Scientia. Série: Ciências da Saúde, Santa Maria, v. 13, n. 2, p. 247-263, 2012. Disponível em:

https://www.periodicos.unifra.br/index.php/disciplinarumS/article/viewFile/1018/963

NARCISO, Fernanda Veruska; MELLO, Marco Túlio. Segurança e saúde dos motoristas profissionais que trafegam nas rodovias do Brasil. Rev. Saúde Pública. v.51, 2017. Disponível em: http://www.scielo.br/scielo.php?pid=S0034- 
$89102017000100604 \&$ script=sci_arttext\&tIng=pt

NASCIMENTO, Eurípedes Costa; NASCIMENTO, Evania; SILVA, José de Paula. Uso de álcool e anfetaminas entre caminhoneiros de estrada. Rev. Saúde Pública. v. 41, n. 2, p. 290-293, 2007. Disponível em:

http://www.scielo.br/scielo.php?script=sci_arttext\&pid=S0034-89102007000200017

OLIVEIRA, Lúcio Garcia. et al. A continuidade do uso de anfetaminas por motoristas de caminhão no Estado de São Paulo, Brasil, a despeito da proibição de sua produção, prescrição e uso. Cad. Saúde Pública. v. 29, n. 9, p.1903-1909, 2013. Disponível em: http://www.scielo.br/scielo.php?script=sci_arttext\&pid=S0102-311X2013000900028

OLIVEIRA, Lúcio Garcia. et al. Condições ocupacionais e o risco de uso de anfetaminas entre motoristas de caminhão. Rev Saúde Pública. v. 49, n.61, 2015. Disponível em: http://www.scielo.br/pdf/rsp/v49/pt_0034-8910-rsp-S0034-89102015049005944.pdf

Prefeitura de Montes Claros - MG Aspectos Gerais. Disponível em: http://www.montesclaros.mg.gov.br/cidade/aspectos_gerais.htm

SILVA, Luna Gonçalves. et al. Vínculos empregatícios, condições de trabalho e saúde entre motoristas de caminhão. Revista Psicologia Organizações e Trabalho. v. 16, n. 2, p. 153-165. Disponível em: https://dx.doi.org/10.17652/rpot/2016.2.675

SILVA-JÚNIOR, Francisco Pereira. et al. Risk factors for depression in truck drivers. Soc Psychiatry Psychiatr Epidemiol. v. 44, n. 2, p.125-129, 2009. Disponível em: http://repositorio.unifesp.br/handle/11600/31280

SINAGAWA, Daniele Mayumi. et al. Association between travel length and drug use among Brazilian truck drivers. Traddic Inj Prev. v. 16, n. 1, p. 5-9, 2015. Disponível em: https://www.ncbi.nlm.nih.gov/pubmed/24697351

SOUZA, José Carlos; PAIVA, Tereza; REIMÃO, Rubens. Sleep habits, sleepiness and accidents among truck drivers. Arq Neuropsiquiatr. v. 63, n. 4, p. 925-930, 2005. Disponível em: http://www.scielo.br/scielo.php?script=sci_arttext\&pid=S0004282X2005000600004

TAKITANE, Juliana. et al. Uso de anfetaminas por motoristas de caminhão em rodovias do Estado de São Paulo: um risco à ocorrência de acidentes de trânsito? Ciênc. saúde coletiva. v. 18, n. 5, p. 1247-1254, 2013. Disponível em:

http://www.scielo.br/scielo.php?script=sci_arttext\&pid=S1413-81232013000500009

WENDLER, Emerson Augusto; BUSATO, César Roberto; MIYOSHI, Edmar. Uso de anfetaminas por motoristas de caminhão para reduzir o sono. UEPG Ci. Biol. Saúde. v. 9, n. 3/4, p. 7-13, 2003. Disponível em:

http://www.revistas2.uepg.br/index.php/biologica/article/view/364

WILLIAMSON, Ann. Predictors of psychostimulant use by long-distance truck drivers. Am J Epidemiol. v. 166, p. 1320-1326, 2007. Disponível em:

https://www.ncbi.nlm.nih.gov/pub 